\title{
Rabaska
}

Revue d'ethnologie de l'Amérique française

\section{« Une fois, c'était un jeune ethnologue... » Marius Barbeau et les contes populaires}

\section{Ronald Labelle}

Volume 13, 2015

Présence de Marius Barbeau : l'invention du terrain en Amérique

française. Autour d’un legs centenaire (1914-2014)

URI : https://id.erudit.org/iderudit/1033748ar

DOI : https://doi.org/10.7202/1033748ar

Aller au sommaire du numéro

Éditeur(s)

Société québécoise d'ethnologie

ISSN

1703-7433 (imprimé)

1916-7350 (numérique)

Découvrir la revue

Citer cet article

Labelle, R. (2015). « Une fois, c'était un jeune ethnologue... » Marius Barbeau et les contes populaires. Rabaska, 13, 43-54. https://doi.org/10.7202/1033748ar d'utilisation que vous pouvez consulter en ligne. 


\section{Barbeau et l'oralité : le conte}

\section{«Une fois, c'était un jeune ethnologue... » Marius Barbeau et les contes populaires}

Ronald LABELLE

Cape Breton University

\section{Le parcours de Barbeau}

On a souvent raconté comment la rencontre de Marius Barbeau avec l'anthropologue américain d'origine allemande Franz Boas a été déterminante dans son orientation future. Barbeau lui-même, dans un texte d'importance fondamentale publié par la Société royale du Canada en 1916, décrit ainsi sa découverte des contes :

Or, il y a deux ans, à un congrès anthropologique, un savant américain demandait : « Les Canadiens-français, eux, ont-ils conservé leurs traditions orales et leurs contes? » Personne ne put répondre à cette question, faute de documents. L'auteur de ce document s'engagea à faire des recherches. La littérature canadienne écrite offrit une réponse négative, les récits assez nombreux qu'on y lit étant pour la plupart des anecdotes ou tableaux de mœurs fabriqués de toutes pièces par les écrivains. Une conclusion plus satisfaisante, toutefois, s'imposa à la suite d'une visite faite à quelques vieux paysans des environs de Québec. Des contes de fées, de magiciens, d'animaux parlants, de princes amorphosés (métamorphosés), de Petit-Jean, de Parlafine ? Oui, on en savait! Et près de trente contes, récités à Lorette, furent sans retard pris à la sténographie, en août, $1914 .{ }^{1}$

L'enquête qui a suivi a été très féconde. Au total, 245 contes ont été recueillis entre 1914 et $1918^{2}$. Barbeau n'a certainement pas été le plus prolifique des collecteurs de contes : Luc Lacourcière, dont la cueillette s'est étendue sur une plus grande période, en a recueilli près

1. Marius Barbeau, « Le Folklore canadien-français », Mémoires de la Société Royale du Canada, section 1, série 3, vol. 9, mars 1916, p. 450-451.

2. Jean-Pierre Pichette, «"La Sereine de mer" et "Les Bossus". Marius Barbeau et l'édition des contes populaires », Cahiers Charlevoix, n 4, 2000, p. 258. 
de deux mille, soit huit fois plus. Même Conrad Laforte, le spécialiste de la chanson traditionnelle, a recueilli à lui seul plus de cinq cents $\operatorname{contes}^{3}$, mais Barbeau, le pionnier, est celui qui a ouvert la voie aux autres, non seulement en développant des normes pour la cueillette et la transcription des contes, mais en multipliant les efforts pour assurer leur diffusion par l'imprimé. C'est de cet aspect de son œuvre qu'il sera surtout question ici.

\section{La diffusion des contes par l'écrit}

C'est par le biais du Journal of American Folk-Lore ${ }^{4}$, une publication de 1'American Folk-Lore Society, que Barbeau fit d'abord paraître ses contes. De 1916 jusqu'à 1950, huit numéros de la revue furent consacrés aux contes recueillis au Québec par Barbeau et ses collaborateurs, dont Gustave Lanctôt, Évelyn Bolduc, Pierre Daviault, Adélard Lambert et Carmen Roy. Les quatre premières séries de contes parurent entre 1916 et 1923, à l'époque où Franz Boas dirigeait la revue. Boas, qui encourageait fortement la recherche sur le conte au Canada français, était très favorable à la diffusion des résultats d'enquêtes. Mais l'American Folk-Lore Society n'avait pas les sous nécessaires pour soutenir cette initiative et Barbeau a dû financer en partie la publication des premières séries de «Contes populaires canadiens » en fondant des sections canadiennes de la société, obtenant ainsi des cotisations des membres. Une branche québécoise fut mise sur pied en 1915, suivie par la succursale ontarienne en $1918^{5}$.

Barbeau obtint aussi l'appui du gouvernement québécois, ce qui lui a permis de planifier la publication annuelle d'un numéro canadienfrançais de la revue à partir de $1916^{6}$. Son projet ambitieux a connu un succès initial, le Journal of American Folk-Lore consacrant des numéros entiers au folklore du Canada français en 1916, 1917, 1919 et $1920^{7}$. Ces numéros contenaient les trois premières séries de «Contes populaires canadiens », en plus d'un recueil de légendes. Cinq autres séries de contes paraîtront en 1923, 1926, 1931, 1940 et 1950.

Marius Barbeau a aussi publié dans les Mémoires de la Société royale du Canada un article intitulé « Les Métamorphoses dans les

3. Jean Du Berger, « Marius Barbeau, le conte et le conteur », Études françaises, vol. 12, $\mathrm{n}^{\text {os }} 1-2$, avril 1976, p. 70 .

4. La revue portera le titre Journal of American Folklore à partir de 1940.

5. Jean-Pierre Pichette, op. cit., p. 279.

6. Marius Barbeau, « Contes populaires canadiens », Journal of American Folk-Lore, vol. 29, $\mathrm{n}^{\circ} 111,1916$, p. 2.

7. Journal of American Folk-Lore, vol. 29, no 111, 1916, p. 1-154; vol. 30, n 115, 1917, p. 1-160; vol. $32, \mathrm{n}^{\circ} 123,1919$, p. 1-184 ; vol. 33, nº 129, 1920, p. 173-297. 
contes populaires canadiens $»^{8}$, où il fournit de multiples exemples de transformations magiques dans les contes merveilleux recueillis au Québec, mais, après la parution de cette étude en 1916, il a accordé peu d'attention à l'analyse du répertoire. Barbeau a aussi recueilli très peu de contes au Québec après 1918. D'après un relevé complété par Jean-Pierre Pichette, seulement cinq contes se seraient ajoutés au total ${ }^{9}$.

Plusieurs facteurs semblent avoir éloigné Barbeau de la collecte de contes. D'une part, le Musée national du Canada, où il œuvrait en tant qu'anthropologue depuis 1911, ne lui apportait pas l'appui nécessaire à ses projets d'enquête. Dans ses Mémoires inédits cités par Jean-Pierre Pichette, Barbeau dit même qu'en 1925, le nouveau directeur du Musée tenta d'interdire la poursuite de ses activités de recherche en lui rappelant qu'il avait comme tâche d'étudier uniquement les Amérindiens ${ }^{10}$. Étant donné que son employeur s'opposait à toute recherche sur le folklore du Canada français, il était très difficile pour Barbeau d'accorder le temps nécessaire à la collecte de traditions orales et, de plus, il devait souvent financer lui-même ses déplacements. D'autre part, le plus important facteur qui expliquerait son désintérêt envers la cueillette des contes après 1918 serait peut-être le fait que Barbeau a grandement élargi son champ d'intérêt. Il est devenu passionné de chanson folklorique, d'artisanat et de culture matérielle en général, ce qui est attesté par des centaines de publications.

Marius Barbeau a donc dû faire des choix et il a décidé de consacrer beaucoup plus d'attention à la chanson qu'au conte. Andrew Nurse, qui a analysé son parcours scientifique, a conclu que Barbeau avait l'ambition de prolonger l'œuvre commencée avec les Chansons populaires du Canada d'Ernest Gagnon (1865), la seule source importante de chansons largement diffusée pendant les premières décennies du $\mathrm{XX}^{\mathrm{e}}$ siècle $^{11}$. Il a finalement publié son Romancero du Canada en 1937 et plusieurs autres recueils ont suivi mais, comme l'a démontré Nurse, la réalisation matérielle d'un livre était toujours un défi et ses projets d'édition ont nécessité des années. Il lui aura ainsi fallu six ans pour mener à terme le projet de Romancero du Canada, car les maisons d'édition approchées n'osaient pas affecter le financement nécessaire

8. Marius Barbeau, «Les Métamorphoses dans les contes populaires canadiens », Mémoires de la Société royale du Canada, section 1, série 10, vol. 10, 1916, p. 143-160.

9. Jean-Pierre Pichette, op. cit., p. 258.

10. Ibid., p. 277.

11. Andrew Nurse, " "The Best Field for Tourist Sale of Books" : Marius Barbeau, the Macmillan Company, and Folklore Publishing in the 1930s », Papers of the Bibliographic Society of Canada / Cahiers de la Société bibliographique du Canada, vol. 36, n 1, 1998, p. 11. 
et les demandes d'aide de Barbeau auprès du gouvernement du Québec s'étaient avérées infructueuses ${ }^{12}$.

Barbeau a donc consacré la plupart de ses efforts à d'autres domaines que le conte après la parution, dans le Journal of American Folk-Lore, des deux premières séries de «Contes populaires canadiens » en 1916 et 1917. Il a toutefois ouvert la voie aux chercheurs comme Évelyn Bolduc et Gustave Lanctôt qui ont continué la série. Barbeau avait écrit en 1917 que les textes publiés dans le Journal of American Folk-Lore étaient de nature « scrupuleusement et sèchement scientifique », ajoutant qu' " on n'y fait nul sacrifice au goût populaire ou à l'art pour l'art ${ }^{13} »$. Barbeau avait noté les contes en utilisant sa technique personnelle de sténographie, adaptée à partir de méthodes existantes. Il avait donc la possibilité de publier des textes qui reproduisaient assez fidèlement les témoignages oraux recueillis en enquête ${ }^{14}$. Les autres collecteurs qui ont suivi se sont inspirés du modèle établi par Barbeau, cherchant à présenter des textes qui conservaient un style oral.

Les textes de contes publiés dans le Journal of American FolkLore répondaient bien aux critères scientifiques des responsables de la revue, mais s'adressaient à un public limité. Au cours des années suivantes, lorsque Barbeau décida de mettre entre les mains du public les bijoux du répertoire folklorique chanté, il eut aussi l'idée de remettre en circulation les contes traditionnels, dont la transmission orale était en déclin.

\section{Les ouvrages destinés au grand public}

Marius Barbeau a finalement publié plusieurs recueils de contes visant le grand public, mais ces ouvrages contrastent nettement avec ceux consacrés aux chansons, car il s'agissait, avec une seule exception, de bouquins très modestes s'adressant principalement aux enfants. Afin de comprendre cette orientation, il faut se rappeler que Barbeau était toujours conscient des difficultés du marché de l'édition. Alors qu'il avait eu l'ambition de publier une grande anthologie de la chanson traditionnelle, dont le Romancero du Canada ne serait que le premier volume $^{15}$, ses ambitions dans le domaine du conte étaient beaucoup plus modestes, suggérant que Barbeau n'y voyait pas un grand potentiel commercial. Pendant les années 1930 et 1940, il produisait des manus-

12. Ibid., p. 12-15.

13. Ibid., p. 10.

14. Jean-Pierre Pichette, op. cit., p. 280-282.

15. Voir Andrew Nurse, op. cit., p. 15 et 23. 
crits en quantité et cherchait par tous moyens de les faire éditer. Il faut se rappeler que le conte populaire n'était qu'un des nombreux domaines qui retenaient son attention. En 1935, par exemple, il rapporta qu'il avait six livres sous presse et huit autres déjà soumis à des éditeurs ${ }^{16}$. Plusieurs de ces manuscrits portaient sur les arts anciens et l'artisanat traditionnel, mais on y trouvait aussi ses premiers recueils de contes.

Deux modestes recueils de contes paraissent en 1935, Il était une fois et Grand'mère raconte, contenant chacun dix récits. Un troisième intitulé Les Rêves des chasseurs paraîtra en 1942, cette fois-ci avec neuf contes ${ }^{17}$. Tous ces textes présentaient des adaptations de contes parus dans le Journal of American Folk-Lore. Au total, quinze textes provenaient des quatre premières séries de «Contes populaires canadiens », dont neuf issus de la première série.

Entre 1950 et 1953, Barbeau publia la série de douze fascicules intitulée Les Contes du Grand-Père Sept-Heures. Il s'agissait de petites brochures contenant au total 49 contes et qui s'adressaient aux enfants ${ }^{18}$. Malgré le caractère très modeste de la série, on y trouve de belles illustrations de l'artiste Arthur Price, un gendre de Barbeau. L'auteur y a aussi inclus en appendice les références aux sources originales des contes dans le Journal of American Folk-Lore, ce qu'il n'avait pas fait dans ses recueils antérieurs.

Reste à signaler un dernier recueil de huit contes en traduction anglaise intitulé The Golden Phoenix and Other French Canadian Fairy Tales ${ }^{19}$. Cet ouvrage est intéressant pour plusieurs raisons. C'est un livre à couverture rigide, abondamment illustré par Arthur Price et agrémenté d'une postface où Barbeau présente les contes, expliquant que certains, dont celui du « Fin voleur », remontent à l'Antiquité. A propos de la traduction par Michael Hornyansky, Barbeau écrit : «Mr Hornyansky and I aimed at achieving in our own way a literary uplifting similar to that of Grimm, Anderson and Perrault in the tales of their people that have now become familiar everywhere. ${ }^{20} " \mathrm{Cet}$ ouvrage connut un succès considérable, ayant été réimprimé au moins cinq fois après 1958, pour enfin faire l'objet d'une nouvelle édition à couverture souple en 1980.

16. Andrew Nurse, op. cit., p. 7.

17. Marius Barbeau, Il était une fois, Montréal, Beauchemin, 1935 ; Grand'mère raconte,

Montréal, Beauchemin, 1935 ; Les Rêves des chasseurs, Montréal, Beauchemin, 1942.

18. Marius Barbeau, Les Contes du Grand-Père Sept-Heures, Montréal, Les éditions Chantecler, 1950 et 1953, 12 vol. en 2 séries.

19. Marius Barbeau et Michael Hornyansky, The Golden Phoenix and Other French Canadian Fairy Tales, Toronto, Oxford University Press, 1958.

20. Ibid., p. 141. 
Les recueils de contes publiés en édition originale française ont aussi connu un bon succès commercial. Une deuxième édition de Les Rêves des chasseurs parut en 1950, Il était une fois a été réédité en 1942 et en 1955, et Grand'mère raconte a été réédité quatre fois entre 1942 et 1952. Mais Barbeau ne réussit pas à attirer l'intérêt de grandes maisons d'édition et devait se contenter de publier ses petits bouquins chez Beauchemin, une maison d'édition qui manquait de rigueur, selon lui. En 1935, il écrit en effet : "Both Granger and Beauchemin are slovenly in all the work they do. ${ }^{21}$ "

\section{Les réalisations de Barbeau et ses limites}

En jetant un regard sur l'ensemble de l'œuvre de Barbeau, on s'aperçoit que beaucoup de ses ouvrages de qualité ont été publiés en anglais. On n'a qu'à considérer ses ouvrages sur la culture matérielle comme I Have Seen Québec, les recueils de chansons comme Jongleur Songs of Old Québec, ainsi que son recueil de contes, The Golden Phoenix and Other Tales from Quebec, sans compter ses nombreux ouvrages sur les cultures autochtones du Canada. Il est intéressant de remarquer aussi que Barbeau a publié Folk-Songs of French Canada avec Edward Sapir en 1925, dix ans avant la parution de son premier recueil de chansons en français et que son roman, The Downfall of Temlaham est paru en 1928, vingt ans avant la version française, Le Rêve de Kamalmouk. De plus, sept ans après avoir publié son recueil de légendes intitulé L'Arbre des rêves, il a réalisé une adaptation anglaise, The Tree of Dreams, où il en modifie sensiblement le contenu, afin de viser un public anglophone.

L'Arbre des rêves nous fournit un cas intéressant permettant de voir comment Barbeau concevait ses œuvres selon le public ciblé. Cet ouvrage paru en 1948 présentait quinze textes légendaires puisés dans diverses sources orales et littéraires. Peu étaient issus des enquêtes de terrain de Marius Barbeau lui-même. Le texte qui a fourni le titre du recueil, par exemple, est une adaptation d'une légende publiée par Philippe-Aubert de Gaspé, père, dans Le Foyer canadien en 1866. Barbeau avoue cependant : « La forme ici donnée est un développement imaginatif et littéraire, qui a connu deux ou trois étapes. ${ }^{22}$ » L'édition anglaise publiée en 1955 présente des versions traduites de seulement six des légendes contenues dans l'édition originale. Les six autres textes que l'on y retrouve sont des portraits de personnages pittoresques du passé qui avaient d'abord été présentés en 1945 à la radio nationale

21. Andrew Nurse, op. cit., p. 14-15. Traduction : Granger et Beauchemin sont tous les deux négligents dans tous leurs travaux.

22. Marius Barbeau, L'Arbre des rêves, Montréal, Les Éditions Lumen, 1948, p. 185. 
de $\mathrm{CBC}$ dans le cadre de l'émission « Canadian Yarns ». Trois de ces portraits avaient d'ailleurs été incorporés dans l'ouvrage intitulé The Kingdom of Saguenay en $1936^{23}$.

On s'aperçoit donc que Barbeau remaniait constamment ses textes selon le public visé. Il faut noter que The Kingdom of Saguenay n'avait pas pris comme point de départ un ouvrage antérieur paru en langue française. Selon Andrew Nurse, il s'agissait d'une commandite visant les touristes américains qui affluaient de plus en plus nombreux dans le comté de Charlevoix. Les compagnies de chemin de fer Canadien National et Canadien Pacifique auraient même aidé à défrayer les coûts des illustrations ${ }^{24}$. L'année suivante, Barbeau publia un autre ouvrage visant le marché touristique, Quebec : Where Ancient France Lingers ${ }^{25}$. Cette fois-ci, une version française intitulée Québec où survit l'ancienne France paraît l'année suivante ${ }^{26}$. Ce dernier ouvrage comprend, en plus de textes sur la culture matérielle et les légendes du terroir, un chapitre consacré aux « chansons d'autrefois ». On ne trouve pourtant pas de contes populaires dans les ouvrages visant principalement le marché touristique. On peut supposer que l'auteur hésitait à les inclure puisque les contes traditionnels à caractère universel n'étaient pas ancrés dans les paysages pittoresques du vieux Québec.

Si Barbeau a mis autant d'efforts à produire des publications de langue anglaise sur la culture québécoise, il ne faut pas supposer que ses motifs étaient exclusivement commerciaux. Dans leur article au sujet de la conception du folklore chez Marius Barbeau, Luc Lacourcière et Carmen Roy, les auteurs Dominique Sarny, Anne-Marie Desdouits et Christine Bricault affirment que Barbeau croyait fermement en une identité pancanadienne et qu'il voulait que ses activités contribuent à créer un sentiment d'unité nationale ${ }^{27}$. Il va donc de soi qu'il cherchait à publier dans les deux langues, afin de créer des liens entre « les deux solitudes » canadiennes.

Il est possible aussi que Barbeau ait simplement choisi de profiter de toutes les opportunités qui se présentaient à lui pour diffuser les résultats de ses recherches, que ce soit en anglais ou en français. Il a été extrêmement prolifique en tant qu'auteur, publiant sans cesse à partir du début de sa carrière au Musée national jusqu'à sa mort à l'âge de 86 ans. Dans le domaine du conte, il s'est efforcé de reprendre bon

23. Marius Barbeau, The Tree of Dreams, Toronto, Oxford University Press, 1955, p. 109-112.

24. Andrew Nurse, op. cit., p. 17-20.

25. Marius Barbeau, Quebec: Where Ancient France Lingers, Toronto, MacMillan Press, 1936.

26. Marius Barbeau, Québec où survit l'ancienne France, Québec, Éditions Garneau, 1937.

27. Christine Bricault, Anne-Marie Desdouits et Dominique Sarny, « La Conception du folklore de trois pionniers Marius Barbeau, Luc Lacourcière et Carmen Roy, Ethnologies, vol. 26, nº 2, p. 23-24. 
nombre de textes qui avaient d'abord paru dans le Journal of American Folk-Lore, pour les rediffuser par tous les moyens possibles à un public élargi. Entre 1933 et 1938, il a publié une trentaine de contes dans les journaux La Presse, Le Droit et Le Nouvelliste, et il en a même publié dans la revue jeunesse de la Croix-Rouge canadienne, The Canadian Red Cross Junior Magazine. Ces contes étaient souvent les mêmes qu'il avait inclus dans ses trois premiers recueils, Grand'mère raconte, Il était une fois et Les Rêves des chasseurs. À titre d'exemple, le conte de « La Chatte blanche » a d'abord fait partie de la première série parue dans le Journal of American Folk-Lore en 1916. Plus tard, ce même conte a été publié à trois reprises dans des journaux (La Presse, septembre 1933, Le Droit, décembre 1934, Le Nouvelliste, décembre 1934). Le conte fait ensuite partie du recueil Les Rêves des chasseurs en 1942 et on le retrouve une dernière fois dans La Revue moderne en octobre 1943.

Compte tenu des très nombreuses initiatives qu'il a prises dans le domaine de l'édition, il peut paraître surprenant que Barbeau n'ait jamais réalisé un ouvrage important en langue française consacré au conte populaire, comme il l'a fait en chansons avec le Romancero $d u$ Canada et Le rossignol y chante. Peut-être considérait-il que le public n'était pas prêt à accueillir un projet du genre. Les douze fascicules des Contes du Grand-Père Sept-Heures parus entre 1950 et 1953 auraient pu facilement être regroupés en deux recueils plus substantiels. En ajoutant les 49 contes contenus dans cette série aux 29 autres contes qui avaient été publiés dans trois recueils antérieurs, on arrive à un total de 78 contes publiés par Barbeau. Cela aurait pu représenter une collection impressionnante, si tous les textes avaient été rassemblés. Barbeau avait d'ailleurs évoqué dans la postface de The Golden Phoenix son ambition de suivre dans la voie tracée par Grimm, Anderson and Perrault ${ }^{28}$. Pourtant, il ne semble jamais avoir proposé la réalisation d'un grand projet d'édition.

Barbeau est donc loin d'avoir fait pour le Canada français un travail d'édition de contes semblable à celui des frères Grimm en Allemagne. L'unique recueil publié en anglais, The Golden Phoenix, aurait pu constituer l'ébauche d'un tel projet. L'ouvrage ne comprend que huit contes, mais son éditeur, la succursale canadienne d'Oxford University Press, a réalisé un produit beaucoup plus soigné que les ouvrages parus en français chez les éditeurs Beauchemin et Chantecler. Il est vrai que Barbeau ne pouvait pas consacrer tous ses efforts à l'édition des contes, comme il menait constamment des travaux parallèles dans

28. Marius Barbeau et Michael Hornyansky, op. cit., p. 141. 
d'autres chantiers. Mais il faut se rappeler aussi qu'il était toujours conscient des exigences du marché de l'édition. Peut-être considérait-il les enfants comme étant ses principaux lecteurs au Canada français, d'où la réalisation d'éditions de contes visant exclusivement un jeune public. Du côté anglophone, le succès remporté par The Golden Phoenix, réimprimé à plusieurs reprises, aurait pu encourager Barbeau à se lancer dans un projet d'anthologie de contes en version anglaise, mais il était déjà âgé de 75 ans quand parut la première édition de cet ouvrage et il n'était plus à l'âge d'entreprendre des projets de grande envergure.

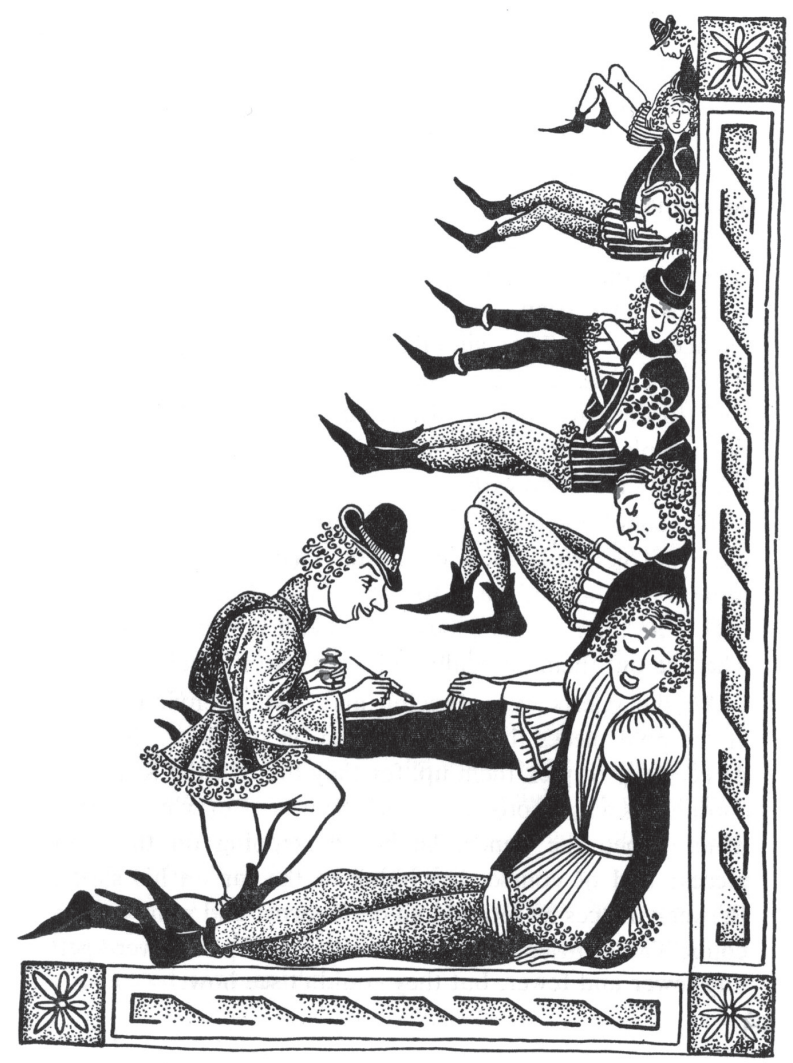

Illustration d'Arthur Price, tirée de The Golden Phoenix, p. 128.

Une dernière observation sur les contes publiés par Barbeau concerne le remaniement des textes oraux. En comparant les versions parues dans le Journal of American Folk-Lore avec celles réimprimées plus tard dans les bouquins pour enfants, on se rend compte que plusieurs textes 
ont été profondément transformés. Une version du conte de Cendrillon, par exemple, avait été publiée sous le titre « Paroles de fleurs, d'or et d'argent » dans le Journal of American Folk-Lore en $1916^{29}$. L'héroïne s'appelait alors «Cendrouillonne », une des nombreuses variantes trouvées dans les versions orales de ce conte. Mais dans la version remaniée qui parut dans Grand'mère raconte ${ }^{30}$, elle s'appelle simplement " Cendrillon », comme chez Perrault. Du coup, le conte perd un peu de sa saveur orale. Dans la postface de The Golden Phoenix, Barbeau avait décrit l'adaptation anglaise de Michael Hornyansky comme étant « a polished retelling », alors que les conteurs de qui ils avaient été recueillis sont qualifiés de simples habitants ${ }^{31}$.

Dans ses adaptations françaises visant le grand public, Barbeau lui-même a «poli » les contes, opérant ainsi une forme d'adaptation littéraire qui les éloignaient de leurs sources orales. Dans son article intitulé «"La Sereine de mer" et "Les Bossus". Marius Barbeau et l'édition des contes populaires », Jean-Pierre Pichette a écrit que, presque sans exception, les textes publiés par Barbeau dans le Journal of American Folk-Lore font preuve d'une " honnête normalisation, davantage soucieuse d'accroître la lisibilité du texte oral, par la clarté du propos et la correction grammaticale, que de réinventer une nouvelle histoire à partir des données populaires. ${ }^{32}$ " $\mathrm{Il}$ en est cependant tout autre dans les livres de contes publiés à partir de 1935 .

Le conte intitulé « Le Rêve des chasseurs » fournit un bon exemple des modifications qu'apportait Barbeau aux textes qu'il présentait au grand public. Dans la première série des « Contes populaires canadiens ", le récit recueilli de madame Prudent Sioui à Lorette commence ainsi :

Il est bon de vous dire qu'une fois c'était trois messieurs et leur cuisinier, qui étaient allés à la chasse, dans les bois. Après avoir chassé toute la journée sans manger, ils n'avaient tué qu'une perdrix. Ils se dirent : « Gardons la perdrix pour le déjeuner. Elle sera à celui qui fera le plus beau rêve. $»^{33}$

Dans le recueil paru en 1942 qui porte le titre du conte, le texte commence plutôt ainsi :

Trois bourgeois et leur cuisinier s'en vont dans les bois, à la chasse. À la fin de la journée, affamés, ils tuent une seule perdrix. Comme c'était bien peu de

29. Marius Barbeau, Journal of American Folk-Lore, vol. 29, n 111, 1916, p. 54-55.

30. Marius Barbeau, Grand'mère raconte, Montréal, Beauchemin, 1935, p. 25-29.

31. Marius Barbeau et Michael Hornyansky, op. cit., p. 141.

32. Jean-Pierre Pichette, op. cit., p. 274.

33. Marius Barbeau, « Contes populaires canadiens », op. cit., p. 134-135. 


\section{Les rêves des chasseurs}

(Illustrations de Phoobé Thompson)
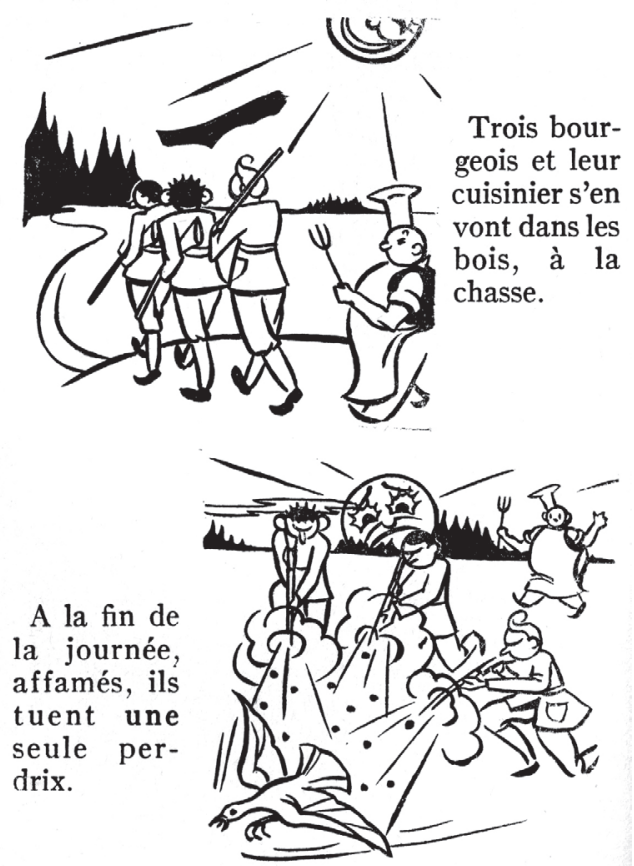

Illustration de Phoebé Thompson, tirée de Les Rêves des chasseurs, p. 8.

chose à se mettre sous la dent, ils disent : « Gardons la perdrix pour le déjeuner. L'aura celui qui fera le plus beau rêve. $»^{34}$

Barbeau supprime la formule d'ouverture «Il est bon de vous dire que... » et il ajoute le commentaire qu'une perdrix, c'est bien peu de chose à se mettre sous la dent. La narration s'éloigne quelque peu du style oral pour prendre un caractère plus littéraire, ce qui est typique dans un grand nombre d'adaptations des contes de Barbeau. On est aussi frappé par l'abondance des illustrations dans l'édition de ce conte. Huit dessins de Phoebé Thompson accompagnent le texte, prenant plus d'espace que le conte lui-même ${ }^{35}$.

Dans l'introduction du recueil de légendes intitulé L'Arbre des rêves, Marius Barbeau cite Russel K. Alspack qui écrivait : " Le folklore n'a pas de meilleure fin que d'inspirer ses poètes et ses prosateurs ${ }^{36} »$.

\footnotetext{
34. Marius Barbeau, Les Rêves des chasseurs, op. cit., p. 8-9.

35. Ibid., p. 8-11.

36. Marius Barbeau, L'Arbre des rêves, op. cit., p. 7.
} 
Un peu comme les auteurs québécois du XIX ${ }^{\mathrm{e}}$ siècle, Barbeau rêvait d'une littérature canadienne qui serait fortement inspirée d'histoires issues du terroir et, malgré le rôle qu'il jouait dans le monde scientifique en tant qu'anthropologue rattaché au Musée national du Canada, Barbeau a finalement tenté lui-même de contribuer à cette littérature en produisant quelques œuvres de création, mais surtout en réalisant un nombre assez important d'adaptations de contes et de légendes. 